\title{
Are squid statoliths hollow during embryogenesis?
}

R. Rosa*, G. Dionísio***, A. Bensimon-Brito*******, A Jacinto,.*******, M.S. Pimentel*, K. Trübenbach*, A. Moreno*****, P. Barquinha****** and M. Diniz *******

*Laboratório Marítimo da Guia, Centro de Oceanografia, Faculdade de Ciências da Universidade de Lisboa, Av. Nossa Senhora do Cabo, 939, 2750-374 Cascais, Portugal.

**Departamento de Biologia \& CESAM, Universidade de Aveiro, Campus Universitário de Santiago, 3810-193 Aveiro, Portugal

***Centro de Estudos de Doenças Crónicas, CEDOC, Faculdade de Ciências Médicas, Universidade Nova de Lisboa, Portugal

****Instituto Gulbenkian de Ciência, Rua Quinta Grande 6 2780-156 Oeiras, Portugal

***** Instituto Português do Mar e da Atmosfera, Avenida de Brasília, 1449-006 Lisboa, Portugal

******CENIMAT/I3N e Departamento de Ciências dos Materiais, Faculdade de Ciências e Tecnologia, Universidade Nova de Lisboa, Quinta da Torre, 2829-516 Caparica, Portugal

*******REQUIMTE, Departamento de Química, Centro de Química Fina e Biotecnologia, Faculdade de Ciências e Tecnologia, Universidade Nova de Lisboa, Quinta da Torre 2829-516, Caparica, Portugal.

Squid statoliths are paired calcareous concretions essentially composed of calcium carbonate crystallised as aragoniteand found inside cranial fluid-filled cavities, the statocysts - the main organ of detection of gravity and movement. Statolith use as an ageing tool was validated in the 1980s, when the daily periodicity of the growth increments was confirmed by chemically marking these structures in animals maintained in aquaria [1,2]. Although statolith analysis has brought major advances in the knowledge of squid population-dynamics, little is known about the processes involved in statolith's calcification and increment deposition (e.g. during embryogenesis). According to Villanueva (2000), embryonic statolith development involves the formation of structures such as the focus, nucleus, postnuclear zone and natal ring.

The aim of this study was to investigatethe microstructure of the embryonic statolith, considering all main structures, in recently-hatched squids using optical (fluorescence) microscopy, scanning electron microscopy (SEM) and confocal microscopy.

Recently-spawned egg masses of the European squid, Loligo vulgaris, were collected in the west coast of Portugal. After hatching at $13-15^{\circ} \mathrm{C}$, statoliths were removed from the paralarvae andpreserved at $-20^{\circ} \mathrm{C}$. Prior to confocal analysis, statoliths were submitted to Alizarin red S (ARS) staining ( $5 \%$ of ARS in $0.1 \% \mathrm{KOH}$ ) to verify the presence of calcium content. In addition, images from optical microscopy and scanning electron microscopy (SEM) were acquired.

The findings obtained by auto-fluorescence observation (Figure 1 A, B) and SEM (Figure 1 C), show that the embryonic statolith is hollow in some of the area between the nucleus and the natal ring. ARS stained samples analysed in confocal microscopy (Figure $2 \mathrm{~A}-\mathrm{G}$ ), confirmed the previous observation.

This surprising structural feature in the origin of $\mathrm{CaCO}_{3}$ sensorial structures has never been described before and suggests a production, with minimum of material and energy expenditure. This is fundamentally interesting not only in biological terms but also considering biotemplating orbiomimetics approaches (i.e. synthetic approaches), where hollow calcium carbonate structures have attracted considerable attention owing to their unique structural, optical, and surface properties that lead them to a wide range of applications, including as templates for functional architecture composite materials [3].

References

1. Villanueva R., Mar. Biol., 137:161-168, 2000.

2. Arkhipkin A., Mar. Freshw. Res., 56:573-583, 2005.

3. Walsh D. and Mann S., Nature, 377:320-323, 1995. 


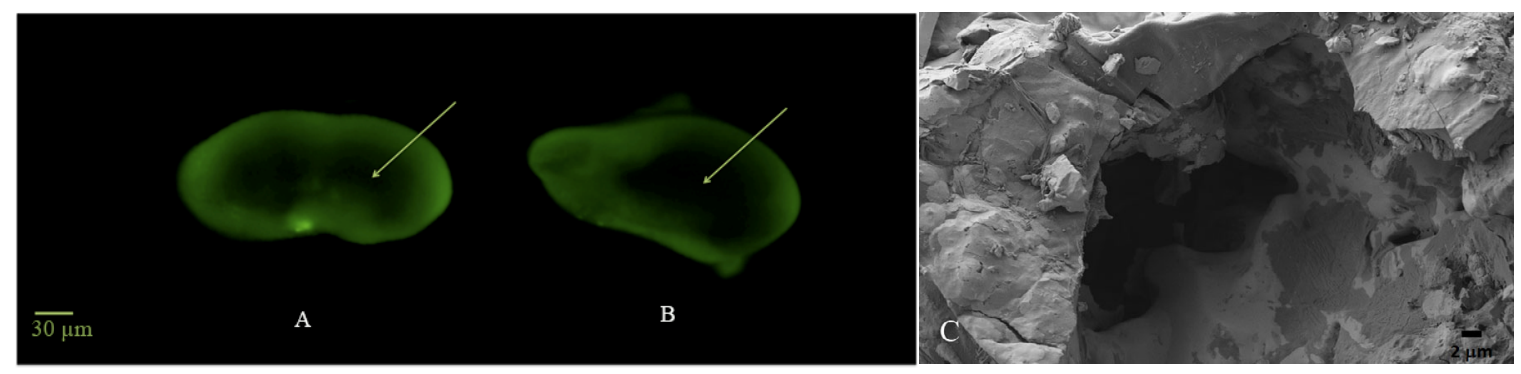

Fig. 1-(A, B).Statoliths of recently-hatched squid (Loligo vulgaris) observed under optical microscopy (autofluorescence) (images from different embryonic statoliths). Scale bar $=30 \mu \mathrm{m}$. Green arrows indicate hollow structure. (C). Transversal cut SEM imageof hollow statolith (Loligo vulgaris). Scale bar $=2 \mu \mathrm{m}$.

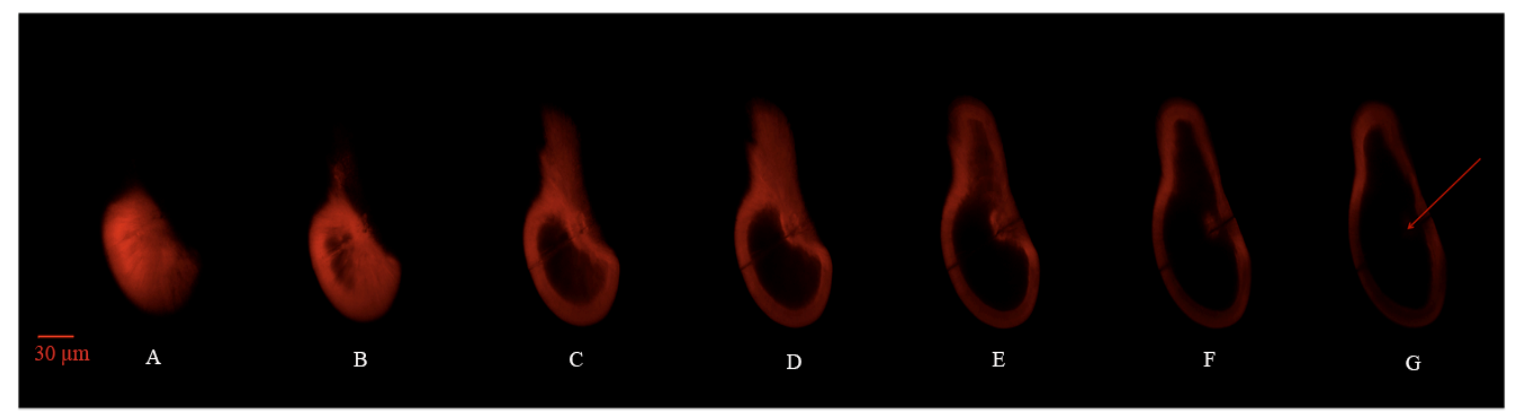

Fig. 2 -Statolith of recently-hatched squid (Loligo vulgaris) observed under confocal microscopy (images from the side (A) to middle (G) of the embryonic statolith). Scale bar $=30 \mu \mathrm{m}$. Red arrow indicates hollow structure.

The Portuguese Foundation for Science and Technology (FCT) supported this study through project grant PTDC/BIA-BEC/103266/2008 to R. Rosa. 